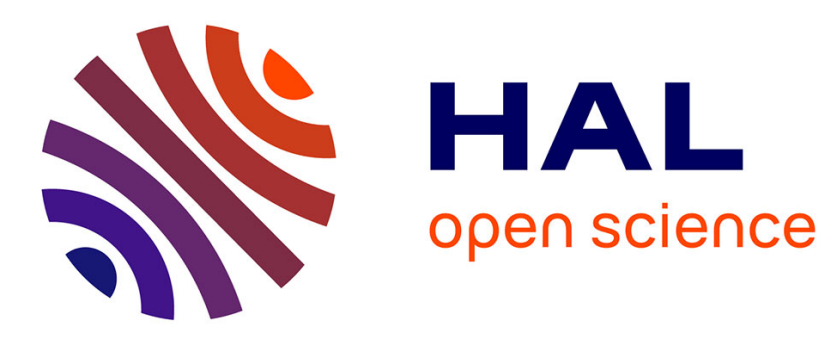

\title{
In-plane elasticity controls the full dynamics of red blood cells in shear flow
}

Simon Mendez, Manouk Abkarian

\section{To cite this version:}

Simon Mendez, Manouk Abkarian. In-plane elasticity controls the full dynamics of red blood cells in shear flow. Physical Review Fluids, 2018, 3 (10), 10.1103/PhysRevFluids.3.101101 • hal-01949575

\section{HAL Id: hal-01949575 \\ https://hal.science/hal-01949575}

Submitted on 10 Dec 2018

HAL is a multi-disciplinary open access archive for the deposit and dissemination of scientific research documents, whether they are published or not. The documents may come from teaching and research institutions in France or abroad, or from public or private research centers.
L'archive ouverte pluridisciplinaire HAL, est destinée au dépôt et à la diffusion de documents scientifiques de niveau recherche, publiés ou non, émanant des établissements d'enseignement et de recherche français ou étrangers, des laboratoires publics ou privés. 


\title{
In-plane elasticity controls the full dynamics of red blood cells in shear flow
}

\author{
Simon Mendez ${ }^{1}$ and Manouk Abkarian ${ }^{2}$ \\ ${ }^{1} I M A G$, CNRS, Univ Montpellier, Montpellier, France \\ ${ }^{2} C B S$, CNRS, INSERM, Univ Montpellier, Montpellier, France
}

\begin{abstract}
The problem of understanding the movement of red blood cells (RBCs) is at the heart of hemorheology. It has thus motivated an extensive body of experimental and numerical works, which showed that RBCs display a rich dynamical behavior in pure shear flow. However, a clear physical understanding of the coupling between cells orientation, membrane deformations and circulation is still not emerging, notably due to the lack of a comprehensive and tractable model to serve as the theoretical foundations for data analysis. Here, we propose a new low-order model which, combined with detailed simulations and existing experimental data, demonstrates how membrane in-plane deformations and elasticity are the essential ingredients responsible for RBCs dynamics at low shear stresses. Our approach demonstrates that out-of-plane deformations and fluid inertia are not necessary to explain the RBC dynamics and underlines the importance of membrane stress-free shape. By reproducing all the details of known RBCs dynamics in shear flow, the new low-order provides a single framework to understand the full dynamics of RBCs at low shear stresses.
\end{abstract}

Red blood cells (RBCs) are the essential oxygen carriers of our body and compose almost half of blood's entire volume. Understanding their behavior in flow is therefore a key step to characterize both physiological and pathological blood circulatory conditions, and to propose non-invasive diagnostic tools by testing their impaired dynamics. The apparent structural simplicity of RBCs contrasts with the complexity of predicting their dynamics [1. Indeed, a RBC is a micron-scale deformable bag, of the shape of a biconcave disk at rest, containing a Newtonian solution of hemoglobin of viscosity $\eta_{i}$, typically five times the viscosity of water and higher than the outer viscosity $\eta_{o}$ in physiological conditions. This solution is enclosed by a thin composite membrane made of an incompressible fluid lipid bilayer, scaffolded inside by a two-dimensional network of elastic filaments of spectrin 2] with a typical in-plane shear modulus $G_{s}$. Understanding the dynamics of a RBC comes down to two main issues: how its shape adapts to viscous stresses and how the resulting internal flow impacts cell behavior. This fluid-structure problem is highly non-linear and non-local in nature and despite decades of research, the full phase diagram of the different dynamical states of a RBC in pure shear flow is still not available, as demonstrated by the recent discovery of new dynamical states observed in physiological conditions of strong flows 3 .

However, experiments realized during the last decades on single RBCs for shear stresses $\eta_{o} \dot{\gamma}$ lower than 0.1 $\mathrm{Pa}$ 4, 5] identified numerous dynamics of orientation where membrane out-of-plane deformations are small and cells maintain their discocyte shape. For instance, RBCs present a dual solid-liquid behavior for increasing $\eta_{o} \dot{\gamma}$, depending on the viscosity ratio $\lambda=\eta_{i} / \eta_{o}$ [6, 7]. For $\lambda \gtrsim 3$, the cell flips like a solid coin. Its axis of symmetry precesses around the vorticity direction, describing a closed trajectory. However, unlike a solid ellipsoid [8], this orbit progressively drifts for increasing shear rate $\dot{\gamma}$ until the symmetry axis aligns with the vorticity direction: the cell rolls [5, 6, 9, 10]. For $\lambda \lesssim 3$, the orbital drift to rolling is maintained [5, 9, but for higher $\dot{\gamma}$, rolling is not stable and is replaced by a modified droplet-like behavior called swinging 4, 5: the membrane circulates around the center of mass like a tank-tread, while the orientation of one of the cell's largest axis of inertia oscillates around an average angle whose value decreases with $\dot{\gamma}$. A rich corpus of numerical simulations reproduced part of these motions [3, 11 16, but even a simple question as the essential ingredients yielding this complex dynamics remains unanswered. Dupire et al. [5] hypothesized for instance that in-plane elasticity may be responsible for orbital drift, but the only test provided was to rigidify the RBCs, indeed preventing drift but simultaneously precluding in-plane and out-of-plane deformations and inner flow. Whether out-of-plane deformations are necessary to explain RBC dynamics at low shear stress, or solely the viscosity ratio is unknown. Moreover, inertia, which is known to promote rolling motion for solid ellipsoids [17, may play a role. Inertia and out-of-plane deformations are small in this flow regime, but the orbital drift is a very slow process, occurring over hundreds of time units $\dot{\gamma}^{-1}$. This suggests that even small effects may contribute to the long-term dynamics of RBCs.

Important breakthroughs in the physical understanding of RBC dynamics sprung from the development of theoretical models where the axis of symmetry of the cell is bound to the shear plane. Keller and Skalak 18 understood the fundamental role played by $\lambda$ in the transition between flipping and tank-treading. Later, the existence of the membrane stress-free shape different from the sphere was added to this approach [4, 19, inspired by the observations of in-plane elasticity and shape memory for the RBC membrane [20, 21]. These models predicted the transition between flipping and swinging with $\dot{\gamma}[4$. The membrane elements have to overcome an energy barrier to circulate around the RBC and make the cell tanktread/swing. In-plane elasticity was thus identified as a 
key element controlling dynamics for this planar configuration. However, such conclusions have not been generalized to 3-D dynamics: the absence of comprehensive approaches coupling arbitrary orientation, membrane circulation and stress, isolating the role played by the geometry and by each structural element, has been a true limitation for the understanding of RBC dynamics.

In this letter, we propose a three-dimensional low-order model for RBCs dynamics that we compare to detailed simulations and existing experimental data. We demonstrate the essential role played by both membrane inplane deformations and viscosity ratio. Our approach underlines the importance of membrane stress-free shape and reproduces all the dynamics observed both experimentally and numerically, simultaneously ruling out outof-plane deformations and fluid inertia as necessary elements to explain the behavior of RBCs under shear.
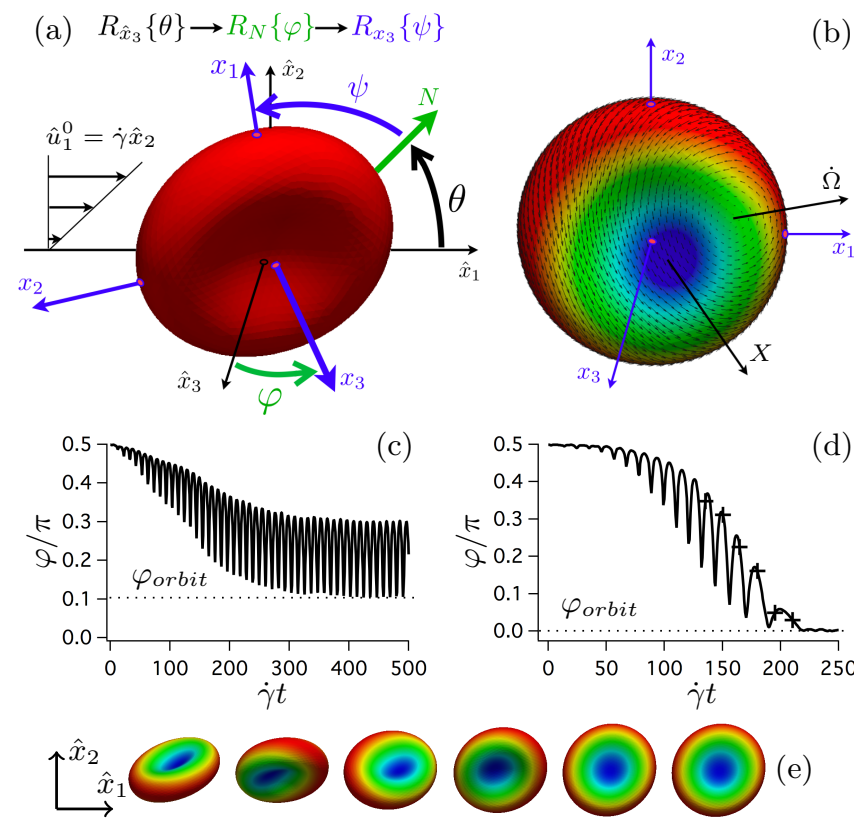

FIG. 1. Geometry and variables of interest $(\mathrm{a}, \mathrm{b})$ and examples of dynamics in shear flow (c-e). (a) The orientation of the body frame $\left(x_{i}\right)$ with respect to the fixed frame $\left(\hat{x}_{i}\right)$ is defined by the Euler angles 44. (b) The membrane tank-treading is defined in the body frame by the tank-treading rate vector $\dot{\Omega}$ and the small axis of the reference shape $X$. The RBC is colored by the reference shape radius (radial distance to the cell center), from blue (low values, small axis) to red (large values, long axes). (c) Simulation of an orbit selection $(\lambda=1$, $\left.C a=0.016, v_{r e f}=0.997\right): \varphi$ along time. The minimum angle in the stationary state, $\varphi_{\text {orbit }}$, characterizes the limit orbit. (d-e) Simulation from flipping to rolling $(\lambda=1, C a=0.025$, $\left.v_{\text {ref }}=0.997\right): \varphi$ along time (d) and corresponding sequence (e) of the simulated RBC colored as in (b). The + in (d) mark the instants when the shapes are displayed in (e).

The problem geometry is shown in Fig. 1. $\hat{x}_{i}$ (unit vectors $\hat{e}_{i}$ ) denote the coordinates of a fixed frame $\hat{\mathcal{R}}$, and $x_{i}$ (unit vectors $e_{i}$ ) the coordinates of a body frame $\mathcal{R}$ of same origin, whose axes correspond to the principal axes of the cell. To define the orientation of the cell with respect to the fixed frame, the Euler angles $\theta, \varphi$ and $\psi$ are used (Fig. 1(a) and supplementary material [44]). An external shear flow is imposed: $\hat{u}_{1}^{0}=\dot{\gamma} \hat{x}_{2}$. The relative strength of the shear flow is defined by the capillary number $C a=\eta_{o} \dot{\gamma} a / G_{s}$, with $a=2.82 \mu \mathrm{m}$ a characteristic size of the RBC 12 .

The small axis (symmetry axis) of the RBC is along $x_{3}$. The Euler angle $\varphi$ is the angle between $x_{3}$ and the vorticity direction $\hat{x}_{3}$, and thus characterizes the orbits of the cell. Membrane circulation is tracked in a Lagrangian way following the location of the small axis of the stressfree shape of the cytoskeleton in the body frame, denoted by vector $X$ (Fig. 1 $\mathrm{b}$ ). When $X$ is not along $x_{3}$, the membrane has circulated and stored in-plane elastic energy.

The present analysis is based on models of decreasing complexity. We first present simulations of the full fluidstructure interaction problem, including all modes of deformations and small yet non-zero fluid inertia. Simulations provide reference data for the subsequent low-order modeling. Simulations are performed using YALES2BIO [3, 16, 22, 26] (see supplementary material [44]), a solver for the incompressible Navier-Stokes equation based on the immersed boundary method. The infinitely thin membrane resists shear and area changes following the Skalak model [27]. Bending resistance is modeled by the Helfrich curvature energy [28, 29. The membrane is prestressed at rest, its stress-free shape being a quasispherical oblate ellipsoid [12, 13, 30, 31], with the same surface area as the RBC. Different stress-free ellipsoids are used, defined by their reduced volume $v_{r e f}$, the volume of the ellipsoid divided by the volume of the sphere having the same surface area. This reference shape is first deflated until an equilibrium biconcave shape is reached, which is then subjected to shear. The higher $v_{r e f}$, the lower the elastic energy barrier for membrane circulation [13, 20, 32. In all simulations, $G_{s}=2.5 \mu \mathrm{N} \cdot \mathrm{m}^{-1}$ [31, 33, and the bending modulus is $\kappa_{b}=3.0 \times 10^{-19} \mathrm{~J}[16]$.

As a first result, we show an example of orbit selection in Fig. 11(c: after a transient flipping, the cell initially in the shear plane $(\varphi=\pi / 2)$ reorients and flips over a specific limit orbit, characterized by $\varphi_{\text {orbit }}$, the minimum value of $\varphi$ after reorientation. At higher external stress, the cell may reorient to rolling: the $\mathrm{RBC}$ small axis aligns with the vorticity direction $(\varphi \rightarrow 0$, Fig. 11). During the reorientation, the reference shape circulates along the RBC without major cell deformation (Fig. 17).

The orbital drift of RBCs as a function of $C a$ is first examined for $\lambda=1.0$ (Fig. 2): RBCs flip over orbits of decreasing value of $\varphi_{\text {orbit }}$ when $C a$ increases, until rolling is reached $\left(\varphi_{\text {orbit }}=0\right)$, as seen in experiments [5]. For higher $C a, \mathrm{RBCs}$ return to the shear plane $\left(\varphi_{\text {orbit }}=\pi / 2\right)$ and tank-tread/swing [4, 5. This reorientation from rolling to tank-treading is performed without flipping: the RBC takes a fixed inclination and spins without angle oscillations, a motion called 'frisbeeing' or 'hovering', 
reported in simulations [12, 15] and transiently in experiments with a step increase of shear rate [5].

In Fig. 2, results at $\lambda=1$ show that the stress-free shape of the cell determines the stress at which orbital drift occurs. The energy barrier for membrane circulation being higher at $v_{r e f}=0.96$ than at 0.997 , the reorientation occurs for higher $\mathrm{Ca}$. As a consequence, the frisbee motion disappears. Instead, the RBC buckles [3, 16] and becomes a stomatocyte [16]: at such higher stresses, the whole RBC deforms. Note that results align well with the experiments [5] for $v_{r e f}=0.96$ (Fig. 2, inset).

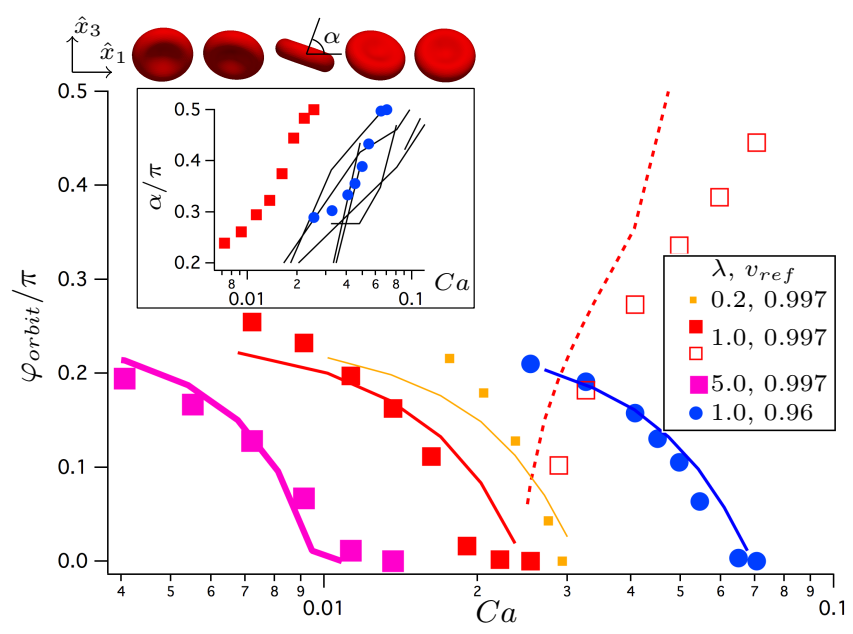

FIG. 2. Orbital drift of a $\mathrm{RBC}$ with $\mathrm{Ca}$ in shear flow varying $\lambda$ and membrane energy barrier. Comparison of $\varphi_{\text {orbit }}$ from simulations (filled symbols) and low-order model (solid lines). The energy barrier is modified in the simulations by changing $v_{\text {ref }}(0.997$ and 0.96$)$ and adjusted in the model (Eq. 3 by changing $C$ (0.00625 and 0.0175 , respectively) based on the data at $\lambda=5$ and $\lambda=1$, respectively. Frisbee angles are also displayed for $v_{r e f}=0.997$ and $\lambda=1$ (open symbols for simulations and dashed line for the model). Inset: angle $\alpha$ between the flow axis and the small axis when the RBC is on the edge while flipping: experiments for different RBCs in PBS-dextran solutions with dextran molecular weight of $10^{5}$ $\mathrm{g} / \mathrm{mol}$ (solid lines) [5] and simulations at $\lambda=1$ (symbols).

The simulations highlight the importance of the membrane stress-free shape [12, 13, 32, and thus its in-plane elasticity, as a key parameter controlling the 3-D dynamics of RBCs. However, the roles of inertia or shape deformations, inherently present both in simulations and in experiments, remain unclear. In order to separate the different mechanisms possibly leading to orbit selection and rolling, we introduce a theoretical model in which inertia and shape deformations are absent. This is inspired by the works of Jeffery [8], Keller and Skalak [18] and their extensions for elastic membranes [4, 19, 32].

The model considers an axisymmetric ellipsoid suspended in an unbounded shear flow $\hat{u}_{1}^{0}=\dot{\gamma} \hat{x}_{2}$, at zero Reynolds number. The internal fluid can flow inside the ellipsoid of prescribed fixed shape. The membrane can circulate along this shape. In the existing models for fluid ellipsoids [4, 18, 19, 32, one principal axis was lying in the shear plane. Here, the orientation is left free to adapt to the flow and the membrane circulation is solved in three dimensions. The tank-treading velocity is defined by an unknown vector $\dot{\Omega}$ around which the membrane circulates (Fig. 1p): the membrane surface velocity $v_{i}^{m}$ in the body frame reads $v_{i}^{m}=a_{i} \epsilon_{i j k} \dot{\Omega}_{j} x_{k} / a_{k}$ [18], where $\epsilon_{i j k}$ is the Levi-Civita symbol in three dimensions and $\dot{\Omega}_{j}$ is the tank-treading rate around axis $x_{j}$.

Since the total moment acting on a freely suspended neutrally buoyant particle vanishes, it may be shown that the spins of the ellipsoid around its axes $\left(\dot{\omega}_{1}=\right.$ $\dot{\theta} \sin \varphi \sin \psi+\dot{\varphi} \cos \psi, \dot{\omega}_{2}=\dot{\theta} \sin \varphi \cos \psi-\dot{\varphi} \sin \psi, \dot{\omega}_{3}=$ $\dot{\theta} \cos \varphi+\dot{\psi}$ ) read:

$$
\left\{\begin{array}{l}
\dot{\omega}_{1}=-\frac{\dot{\gamma}}{2} \sin \varphi \sin \psi+\frac{a_{2}^{2}-a_{3}^{2}}{a_{2}^{2}+a_{3}^{2}} e_{23}^{0}-\frac{2 a_{2} a_{3}}{a_{2}^{2}+a_{3}^{2}} \dot{\Omega}_{1}, \\
\dot{\omega}_{2}=-\frac{\dot{\gamma}}{2} \sin \varphi \cos \psi+\frac{a_{3}^{2}-a_{1}^{2}}{a_{3}^{2}+a_{1}^{2}} e_{31}^{0}-\frac{2 a_{3} a_{1}}{a_{3}^{2}+a_{1}^{2}} \dot{\Omega}_{2}, \\
\dot{\omega}_{3}+\dot{\Omega}_{3}=-\frac{\dot{\gamma}}{2} \cos \varphi
\end{array}\right.
$$

with $a_{1}, a_{2}$ and $a_{3}$ the semi-axes of the ellipsoid $\left(a_{1}=a_{2}\right.$ for the axisymmetric ellipsoid considered). $\dot{\Omega}_{3}$ cannot be distinguished from $\dot{\omega}_{3}$ because $x_{3}$ is an axis of symmetry. The $e_{i j}^{0}$ are the components of the strain rate tensor of the undisturbed flow in the body frame:

$$
\left\{\begin{array}{l}
e_{23}^{0}=\frac{\dot{\gamma}}{2}\left(\frac{1}{2} \cos \psi \sin 2 \theta \sin 2 \varphi+\cos 2 \theta \sin \psi \sin \varphi\right) \\
e_{31}^{0}=\frac{\dot{\gamma}}{2}\left(\frac{1}{2} \sin \psi \sin 2 \theta \sin 2 \varphi-\cos 2 \theta \cos \psi \sin \varphi\right)
\end{array}\right.
$$

The tank-treading rates are obtained by writing the balance between the stress jump across the membrane and the membrane elastic load (calculated assuming that the membrane is a prestressed Kelvin-Voigt viscoelastic material [4, 32]):

$$
\left\{\begin{array}{l}
\dot{\Omega}_{1}=\frac{-2 f_{3}}{f_{2}-\lambda_{e f f} f_{1}}\left[e_{23}^{0}+\frac{\dot{\gamma} \beta C}{C a} \xi_{2} \xi_{3}\right], \\
\dot{\Omega}_{2}=\frac{2 f_{3}}{f_{2}-\lambda_{e f f} f_{1}}\left[e_{31}^{0}+\frac{\dot{\gamma} \beta C}{C a} \xi_{1} \xi_{3}\right] .
\end{array}\right.
$$

The $f_{i}$ and $\beta$ are geometric factors [1, 18]. $\lambda_{e f f}$ is an effective viscosity ratio accounting for membrane viscosity (here we use $\lambda_{\text {eff }}=\lambda$ ). $C$ is a non-dimensional constant accounting for membrane prestress 32] (the larger the reduced volume of the reference shape, the lower $C$ ). $\xi_{i}$ are the coordinates of the small axis of the reference shape mapped on a sphere: $\xi_{i}=X_{i} / a_{i}$ (no summation). $X_{i}$ and thus $\xi_{i}$, are advanced from the tank-treading velocity $v_{i}^{m}$. The model is consistent with existing models for solid and fluid ellipsoids [4, 8, 18. 

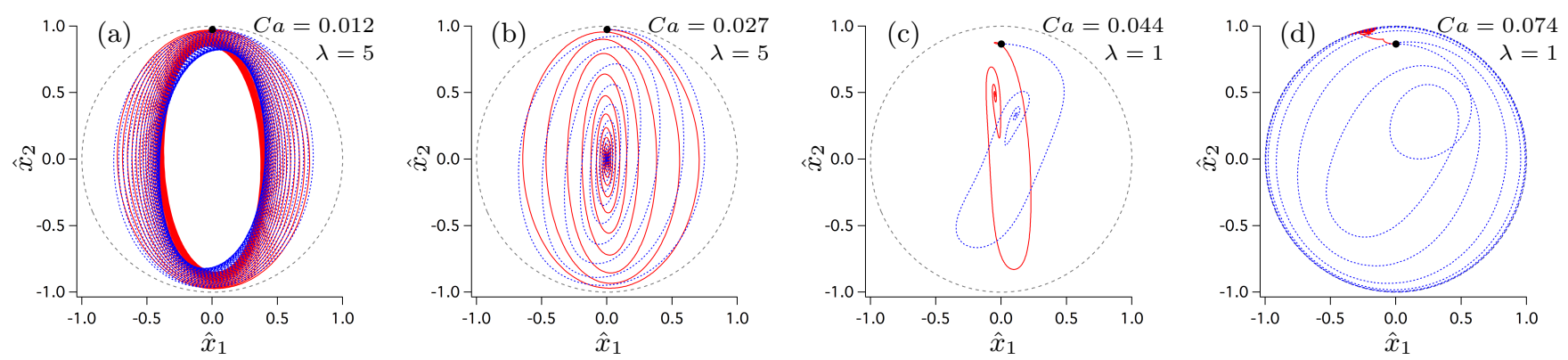

FIG. 3. Dynamics of a fluid ellipsoid $\left(a_{1}=a_{2}=4.2375 \mu \mathrm{m}, a_{3}=1.2511 \mu \mathrm{m}, C=0.01\right)$, in shear flow shown by the trajectories projected in the shear plane of $\left(e_{3}\right)_{\hat{\mathcal{R}}}$ (solid red line) and $(\xi)_{\hat{\mathcal{R}}}$ (blue dotted line). The circle of radius 1 is also shown; black dots mark the initial orientation. Dynamics (see supplementary movies [4]) for different values of $\lambda$ and $C a$ : flipping with orbit selection (a), rolling (b) frisbee (c), transient kayaking and swinging (d).

The system of non-linear ordinary differential equations is solved numerically. The input data are the geometry of the ellipsoid (here, $a_{1}=a_{2}=4.2375 \mu \mathrm{m}$ and $a_{3}=1.2511 \mu \mathrm{m}$ to match the volume and surface of the simulated RBC), $\dot{\gamma}, C a, C, \lambda$ and the initial orientation. Results of the model are displayed in the fixed frame $\hat{\mathcal{R}}$ by projecting on a unit sphere the evolution of the small axes of both the ellipsoid with the vector $\left(e_{3}\right)_{\hat{\mathcal{R}}}$ and of the stress-free shape with the vector $(\xi)_{\hat{\mathcal{R}}}$.

Figure 3 shows that the model reproduces all the typical motions of RBCs reported by experiments and simulations at low shear stress [1]. For $\lambda \gtrsim 3$, the model yields a flipping dynamics over orbits (Fig. 33) determined by $C a$, the orbit shrinking around the vorticity axis when $C a$ increases, until rolling is reached (Fig. $3 \mathrm{~b}$ ). For higher values of $C a$ and $\lambda \gtrsim 3$, rolling is the only stable motion predicted. For $\lambda \lesssim 3$ and increasing $C a$, the model first predicts the flipping and the orbital drift to rolling as for $\lambda \gtrsim 3$, then the frisbee [12, 15] dynamics (Fig. 3c). For higher $\mathrm{Ca}$, the RBC goes to the shear plane to perform swinging 4, possibly with a transient 'kayaking' oscillation around the shear plane (Fig. 3d) [11, 34. In addition to qualitative agreement with the documented behaviors of RBCs, the model aligns well with full simulations (Fig. 2). For $v_{r e f}=0.997$, the prestress coefficient $C$ is adjusted in the model (Eq. 3) using data at $\lambda=5$ and kept constant to predict results at $\lambda=1$ and $\lambda=0.2$. The model is able to predict both the orbital drift and the reorientation from rolling to tank-treading in the frisbee state. The comparison with simulations is excellent, despite some discrepancies, in particular when $C a$ increases. This comparison supports that the in-plane elasticity, the only mechanical property included in the model, is the main effect driving the reorientation. Out-of-plane deformation and inertia only have a second-order effect on the RBC dynamics.

Figures $3(\mathrm{a}-\mathrm{d}$ ) also show the interplay between the dynamics of the ellipsoid and of its membrane: the dynamics depends on the ability of the membrane to circulate over the ellipsoid, which results in the separation between the small axes of the ellipsoid and of the reference shape.
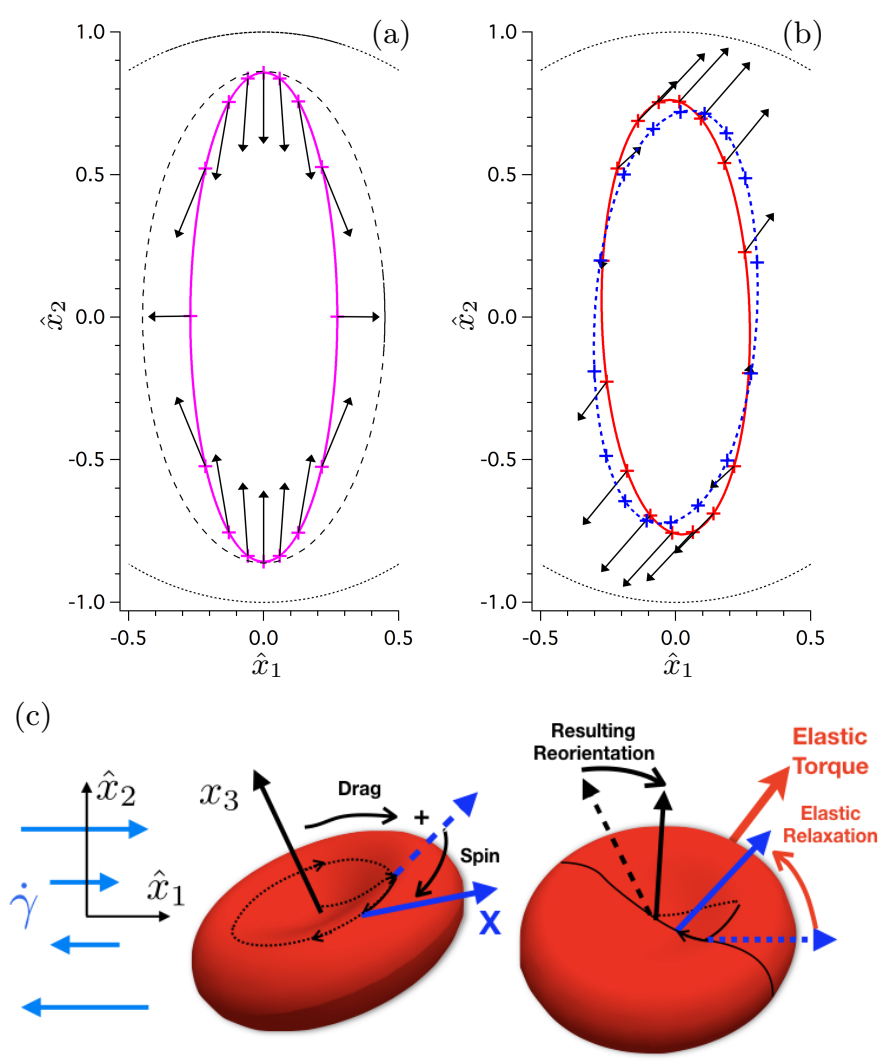

FIG. 4. Example of closed orbits (followed in the clockwise direction) for a fluid ellipsoid in shear flow $\left(a_{1}=a_{2}=4.2375\right.$ $\mu \mathrm{m}, a_{3}=1.2511 \mu \mathrm{m}, C=0.01, \lambda=5$ ), projected in the shear plane. The circle of radius 1 is shown in dotted lines. (a) Cases $C a=0$ (i.e. solid ellipsoid, black dashed line) and $C a=+\infty$ (fluid ellipsoid without membrane elasticity, solid magenta line). Vectors $\dot{\omega}_{\text {Visc.Circ. }}$ are displayed at 16 evenly spaced instants over the period. (b) Case $C a=0.013$ : trajectory of $\left(e_{3}\right)_{\hat{\mathcal{R}}}$ (solid red line) and $(\xi)_{\hat{\mathcal{R}}}$ (blue dashed line). Vectors $\dot{\omega}_{\text {Elast. }}$ are displayed. (c) Schematic of the drift due to the elastic torque.

To further understand the results, we remark that 
Eq. (1) expresses an equilibrium between moments of forces [18. The spins of the ellipsoid result from the sum of the moments of forces exerted by the external flow and those associated to membrane circulation (terms in $\left.\dot{\Omega}_{i}\right)$. The latter can be divided into two contributions (Eq. 3), a purely viscous one and an elastic one, in $1 / C a$. Equation (1) could thus be formally expressed as $\dot{\omega}=\dot{\omega}_{\text {Ext.Flow }}+\dot{\omega}_{\text {Visc.Circ. }}+\dot{\omega}_{\text {Elast. }}$, where the terms of the right-hand side are associated with the external flow, the viscous circulation and the elastic contribution to the circulation, respectively.

Figure 4(a) displays a comparison of the trajectory of the small axis of the ellipsoid in the solid case $(C a=0$, Jeffery orbits) and in the purely viscous case $(C a=+\infty)$. Vectors $\dot{\omega}_{\text {Visc.Circ. }}$ are displayed to analyze how circulation of the membrane exerts an additional moment which modifies Jeffery's trajectory. It is worth noting that torque vectors pointing inwards slow down the trajectory without changing the orbit. On the other hand, torques in the same (resp. opposite) direction as the trajectory deviate the trajectory towards the inside (resp. outside). Figure 4(a) thus shows how the additional moment associated with the circulation of the membrane makes the orbit thinner for $C a=+\infty$. Orbits get actually thinner as $\lambda$ decreases (not shown). Note also that $\dot{\omega}_{V i s c . C i r c .}$ has the same symmetries as $\dot{\omega}_{\text {Ext.Flow }}$.

Figure $4(\mathrm{~b})$ displays the trajectory of $\left(e_{3}\right)_{\hat{\mathcal{R}}}$ and $(\xi)_{\hat{\mathcal{R}}}$ in a case with membrane elasticity. Contrary to $\dot{\omega}_{\text {Visc.Circ. }}$, the elastic torque $\dot{\omega}_{\text {Elast. }}$ is not symmetric with respect to $\hat{x}_{1}$ and $\hat{x}_{2}$ : the orbit thus becomes tilted. It is easily shown that the elastic torque is actually proportional to $\left(e_{3} \cdot \xi\right) e_{3} \times \xi$ : its direction is thus normal to $e_{3}$ and $\xi$, while its norm is determined by the angle between $e_{3}$ and $\xi$, as evidenced by Fig. 4(b). The elastic torque is of course generated by the circulation of the membrane, which is responsible for the storage of in-plane elastic energy. However, a second mechanism determines the direction of the elastic torque: the ellipsoid spins around its symmetry axis (see Eq. 1). This spinning is responsible for a rotation of $\xi$ around $e_{3}$, and consequently of a reorientation of the restoring elastic torque. This is seen in Fig. 4 by the difference in the orientation of $\dot{\omega}_{\text {Visc.Circ. }}$ and $\dot{\omega}_{\text {Elast. }}$. The spinning of the ellipsoid thus makes the membrane restore its elastic energy with a torque oriented differently from the viscous torque which made the membrane circulate. This process is summarized in a schematic way in Fig. 4(c). This element is essential for the understanding of how elasticity controls the dynamics of red blood cells, in particular by modifying their orbits, which are not Jeffery's orbits.

Finally, a full study of the dynamics of RBCs at low shear stresses is made possible by our model: it gathers the different dynamics of RBCs and capsules that were studied before in experiments [5, 9, 10] and simulations [3, 11, 12, 15, 34, 35], but only partially explained until now. By proposing a model able to reproduce the flipping dynamics over orbits, the orbital drift to rolling, frisbeeing, kayaking, swinging in shear flow depending on $\lambda$ and $C a$, we demonstrate that the whole dynamics at low shear rates is mainly controlled by the storage and release of in-plane elastic energy in the membrane.

The behaviors of fluid and rigid ellipsoids are different: Jeffery 8 demonstrated that a rigid ellipsoid rotates indefinitely in shear flow, its symmetry axis precessing around the vorticity direction, describing closed orbits. These orbits are degenerate since an infinite number of them are allowed, their selection depending only on the initial orientation of the particle. This degeneracy, proper to Stokes flows, is removed in real flows. Ellipsoids have been shown to display a rich variety of behaviors depending on both particle and fluid Reynolds numbers [17, 36. For instance, neutrally buoyant oblate ellipsoids have been shown to generally roll on their edge for moderate Reynolds numbers [17, but present a steady inclination for higher inertia. Another cause of orbit selection is the visco-elasticity of the outer fluid [37, 38, though its origin remains unclear. Here, we have identified a new cause for removing the degeneracy of Jeffery's orbits: the energy barrier associated with the circulation of the membrane over the RBC. When the RBC is not in the shear plane, the sliding of its membrane around its ellipsoidal shape generates a lateral torque that makes the particle deviate from Jeffery's orbit.

The role of in-plane elasticity is thus identified and can be now separated from the other effects thanks to our model. We show how the circulation of the stress-free shape coupled to spinning, together with the viscosity contrast, are the two main ingredients to capture the full RBCs phase diagram of movement at low shear stresses. Our model is easy to implement and runs orders of magnitude faster than full simulations. Most importantly, it will allow the stability analysis of the different regimes of movement and a better characterization of their transitions. In addition, the present model paves the way not only to the development of theoretical predictions for blood rheology at low volume fractions, but enables predictions of the dynamics of RBCs in general linear microflows as will be detailed in a full-length publication.

This work was supported by the Labex Numev Convention grants ANR-10-LABX-20. YALES2BIO (http: //www.math.univ-montp2.fr/ yales2bio) simulations have used HPC resources from GENCI-CINES (grants 2016-c2016037194 and 2017-A0020307194). Profs. M. Hillairet, T. Mignon and F. Nicoud (IMAG Montpellier) are gratefully acknowledged for fruitful discussions.

[1] M. Abkarian and A. Viallat, "Fluid-structure interactions in low-Reynolds-number flows," (Royal Society of Chemistry, 2016) Chap. On the importance of red blood 
cells deformability in blood flow, pp. 347-462.

[2] N. Mohandas and P. G. Gallagher, "Red cell membrane: past, present, and future," Blood 112, 3939-3948 (2008)

[3] L. Lanotte, J. Mauer, S. Mendez, D. A. Fedosov, J.-M. Fromental, V. Clavería, F. Nicoud, G. Gompper, and M. Abkarian, "Red cells' dynamic morphologies govern blood shear thinning under microcirculatory flow conditions," Proc. Natl Acad. Sc. USA 113, 13289-13294 (2016)

[4] M. Abkarian, M. Faivre, and A. Viallat, "Swinging of red blood cells under shear flow," Phys. Rev. Lett. 98 (2007), 10.1103/PhysRevLett.98.188302.

[5] J. Dupire, M. Socol, and A. Viallat, "Full dynamics of e red blood cell in shear flow," Proc. Natl Acad. Sc. USA 109, 20808-20813 (2012).

[6] H. L. Goldsmith and J. Marlow, "Flow behaviour of erythrocytes. I. rotation and deformation in dilute suspensions," Proc. R. Soc. Lond. B 182, 351-384 (1972).

[7] T. M. Fischer, M. Stöhr-Liesen, and H. SchmidSchönbein, "The red cell as a fluid droplet: Tank treadlike motion of the human erythrocyte membrane in shear flow," Science 202, 894-896 (1978).

[8] G. B. Jeffery, "The motion of ellipsoidal particles immersed in a viscous fluid," Proc. R. Soc. Lond. A 102, 161-179 (1922).

[9] M. Bitbol, "Red blood cell orientation in orbit $\mathrm{C}=0$," Biophys. J. 49, 1055-1068 (1986).

[10] W. Yao, Z. Wen, Z. Yan, D. Sun, W. Ka, L. Xie, and S. Chien, "Low viscosity Ektacytometry and its validation tested by flow chamber," J. Biomech. 34, 1501-1509 (2001).

[11] D. Cordasco and P. Bagchi, "Orbital drift of capsules and red blood cells in shear flow," Phys. Fluids 25 (2013), $10.1063 / 1.4820472$

[12] D. Cordasco, Yazdani, and P. Bagchi, "Comparison of erythrocyte dynamics in shear flow under different stress-free configurations," $\quad$ Phys. Fluids 26 (2014), $10.1063 / 1.4871300$

[13] Z. Peng, A. Mashayekh, and Q. Zhu, "Erythrocyte responses in low-shear-rate flows: effects of non-biconcave stress-free state in the cytoskeleton," J. Fluid Mech. 742, 96-118 (2014).

[14] K.-I. Tsubota, S. Wada, and H. Liu, "Elastic behavior of a red blood cell with the membrane's nonuniform natural state: equilibrium shape, motion transition under shear flow, and elongation during tank-treading motion," Biomech. Model. Mechanobiol. 13, 735-746 (2014).

[15] K. Sinha and M. D. Graham, "Dynamics of a single red blood cell in simple shear flow," Phys. Rev. E 92 (2015), 10.1103/PhysRevE.92.042710

[16] J. Mauer, S. Mendez, L. Lanotte, F. Nicoud, M. Abkarian, G. Gompper, and D. A. Fedosov, "Complex shapes and dynamics of red blood cells in shear flow under physiological conditions," Phys. Rev. Lett. 121 (2018).

[17] T. Rosén, M. Do-Quang, C. K. Aidun, and F. Lundell, "Effect of fluid and particle inertia on the rotation of an oblate spheroidal particle suspended in linear shear flow," Phys. Rev. E 91 (2015), 10.1103/PhysRevE.91.053017

[18] S. R. Keller and R. Skalak, "Motion of a tank-treading ellipsoidal particle in a shear flow," J. Fluid Mech. 120, 27-47 (1982).

[19] J. M. Skotheim and T. W. Secomb, "Red blood cells and other nonspherical capsules in shear flow: Oscillatory dynamics and the tank-treading-to-tumbling tran- sition," Phys. Rev. Lett. 98 (2007), 10.1103/PhysRevLett.98.078301

[20] T. M. Fischer, C. W. M. Haest, M. Stöhr-Liesen, H. Schmid-Schönbein, and R. Skalak, "The stress-free shape of the red blood cell membrane," Biophys. J. 34, 409-422 (1981).

[21] T. M. Fischer, "Shape memory of human red blood cells," Biophys. J. 86, 3304-3313 (2004).

[22] E. Gibaud, Numerical simulation of red blood cells flowing in a blood analyzer, Ph.D. thesis, Université de Montpellier (2015).

[23] S. Mendez, E. Gibaud, and F. Nicoud, "An unstructured solver for simulations of deformable particles in flows at arbitrary Reynolds numbers," J. Comput. Phys. 256, 465-483 (2014).

[24] J. Sigüenza, S. Mendez, D. Ambard, F. Dubois, F. Jourdan, R. Mozul, and F. Nicoud, "Validation of an immersed thick boundary method for simulating fluid-structure interactions of deformable membranes," J. Comput. Phys. 322, 723-746 (2016).

[25] J. Sigüenza, S. Mendez, and F. Nicoud, "How should the optical tweezers experiment be used to characterize the red blood cell membrane mechanics?" Biomech. Model. Mechanobiol. 16, 1645-1657 (2017).

[26] J. Sigüenza, D. Pott, S. Mendez, S. Sonntag, T. A. S. Kaufmann, U. Steinseifer, and F. Nicoud, "Fluidstructure interaction of a pulsatile flow with an aortic valve model: A combined experimental and numerical study," Int. J. Numer. Meth. Biomed. Eng. 34, e02945:119 (2018).

[27] R. Skalak, A. Tozeren, R. P. Zarda, and S. Chien, "Strain energy function of red blood cell membranes," Biophys. J. 13, 245-264 (1973).

[28] W. Helfrich, "Elastic properties of lipid bilayers: Theory and possible experiments," Z. Naturforsch 28 c, 693-703 (1973).

[29] O. Y. Zhong-can and W. Helfrich, "Bending energy of vesicle membranes: General expressions for the first, second, and third variation of the shape energy and applications to spheres and cylinders," Phys. Rev. A 39, 5280-5288 (1989).

[30] G. H. W. Lim, M. Wortiz, and R. Mukhopadhyay, "Stomatocyte-discocyte-echinocyte sequence of the human red blood cell: Evidence for the bilayer-couple hypothesis from membrane mechanics," Proc. Natl Acad. Sc. USA 99, 16766-16769 (2002).

[31] G. H. W. Lim, M. Wortiz, and R. Mukhopadhyay, "Red blood cell shapes and shape transformations: Newtonian mechanics of a composite membrane," (WILEY-VCH Verlag GmbH \& Co. KGaA, 2008) Chap. 2, pp. 94-269.

[32] J. Dupire, M. Abkarian, and A. Viallat, "A simple model to understand the effect of membrane shear elasticity and stress-free shape on the motion of red blood cells in shear flow," Soft Mat. 11, 8372-8382 (2015).

[33] P. Dimitrakopoulos, "Analysis of the variation in the determination of the shear modulus of the erythrocyte membrane: Effects of the constitutive law and membrane modeling," Phys. Rev. E 85 (2012), 10.1103/PhysRevE.85.041917

[34] C. Dupont, F. Delahaye, B. Barthès-Biesel, and A.-V. Salsac, "Stable equilibrium configurations of an oblate capsule in simple shear flow," J. Fluid Mech. 791, 738757 (2016).

[35] S. Omori, Y. Imai, T. Yamaguchi, and T. Ishikawa, 
"Reorientation of a nonspherical capsule in creeping shear flow," Phys. Rev. Lett. 108 (2012), 10.1103/PhysRevLett.108.138102

[36] T. Rosén, M. Do-Quang, C. K. Aidun, and F. Lundell, "The dynamical states of a prolate spheroidal particle suspended in shear flow as a consequence of particle and fluid inertia," J. Fluid Mech. 771, 115-158 (2015).

[37] F. Gauthier, H. L. Goldsmith, and S. G. Mason, "Particle motions in non-newtonian media. I: Couette flow," Rheol. Acta 10, 344-364 (1971).

[38] G. D'Avino and P. L. Maffettone, "Particle dynamics in viscoelastic liquids," J. Non-Newt. Fluid Mech. 215, 80104 (2015).

[39] J.-M. Charrier, S. Shrivastava, and R. Wu, "Free and constrained inflation of elastic membranes in relation to thermoforming non-axisymmetric problems," The Journal of Strain Analysis for Engineering Design 24, 55-74 (1989).
[40] S. K. Doddi and P. Bagchi, "Lateral migration of a capsule in a plane Poiseuille flow in a channel," Int. J. Multiph. Flow 34, 966-986 (2008).

[41] T. Krüger, B. Kaoui, and J. Harting, "Interplay of inertia and deformability on rheological properties of a suspension of capsules," J. Fluid Mech. 751, 725-745 (2014).

[42] A. Farutin, T. Biben, and C. Misbah, "3D numerical simulations of vesicle and inextensible capsule dynamics," J. Comput. Phys. 275, 539-568 (2014).

[43] A. Guckenberger, M. P. Schraml, P. G. Chen, M. Leonetti, and S. Gekle, "On the bending algorithms for soft objects in flows," Computer Phys. Communications 207, 1-23 (2016).

[44] See Supplemental Material at [URL will be inserted by publisher] for details about the methods and additional animations of the results. 